\title{
Benign Essential Blepharospasm
}

National Institute of Neurological Disorders and Stroke (NINDS)

\section{Source}

National Institute of Neurological Disorders and Stroke (NINDS). Benign Essential

Blepharospasm Information Page.

Benign essential blepharospasm (BEB) is a progressive neurological disorder

characterized by involuntary muscle contractions and spasms of the eyelid muscles. It is a form of dystonia, a movement disorder in which muscle contractions cause sustained eyelid closure, twitching or repetitive movements. BEB begins gradually with increased frequency of eye blinking often associated with eye irritation. Other symptoms may include increasing difficulty in keeping the eyes open, and light sensitivity. Generally, the spasms occur during the day, disappear in sleep, and reappear after waking. As the condition progresses, the spasms may intensify, forcing the eyelids to remain closed for long periods of time, and thereby causing substantial visual disturbance or functional blindness. It is important to note that the blindness is caused solely by the uncontrollable closing of the eyelids and not by a dysfunction of the eyes. BEB occurs in both men and women, although it is especially common in middle-aged and elderly women. 